\title{
Adaptabilidad y cohesión familiar del alumnado de Educación Secundaria Obligatoria
}

\author{
Antonio Urbano Contreras, Lucía Álvarez Blanco y María Teresa Iglesias García
}

Universidad de Oviedo

\section{RESUMEN}

En base a la influencia que el contexto familiar ejerce en el desarrollo personal y en el ámbito escolar, este trabajo busca analizar la percepción que tiene el alumnado de Educación Secundaria Obligatoria (ESO) sobre la adaptabilidad y la cohesión familiar, incluyendo para ello variables sociodemográficas tanto familiares como escolares. Se ha contado con 296 estudiantes (51\% chicos y $49 \%$ chicas), con una edad media de 14,06 años y pertenecientes a los cuatro cursos de esta etapa educativa. El instrumento utilizado para la recogida de información ha sido el FACES II y presentó una buena fiabilidad $(\alpha=0,92)$. Los resultados muestran valores generales positivos en adaptabilidad y cohesión familiar, aunque con necesidades de mejora en aspectos como la disciplina y la participación de los hijos y con matices destacables al considerar las diferencias sociodemográficas (especialmente cuestiones como el curso, haber sido repetidor o la diversidad familiar). Estos resultados permiten identificar áreas prioritarias de intervención y mejora en favor del bienestar del alumnado, así como planificar actuaciones encaminadas a la prevención que repercutan positivamente a nivel personal, familiar y escolar.

Palabras Clave: contexto familiar, educación secundaria, adaptabilidad, cohesión; análisis de necesidades.

\section{Adaptability and family cohesion of students of Compulsory Secondary Education}

\section{ABSTRACT}

According to the influence that the family context tends to have within the school context, this work aims to analyse the adaptability and the family cohesion of the students of Secondary Education, including hence both family and school variables. 296 students participated in this study (51\% boys and $49 \%$ girls), with an average age of 14.06 and belonging to the four grades of this educational stage. The instrument used for gathering the information was the FACES II, presenting a good reliability $(\alpha=0.92)$. The results showed overall positive values in most variables, although with a margin for improvement in aspects like discipline and participation by children, and remarkable nuances considering socio-demographic differences (particularly factors like the grade, having repeated a grade before or the family diversity). These results let us identify priority areas of intervention and improvement in the pursuit of the well-being of the students, and plan preventive actions as well to positively impact at a personal, family, and school level.

Keywords: family context, secondary education, adaptability, cohesion, analysis of needs.

\section{Introducción}

El contexto familiar es un ámbito fundamental para el desarrollo y el bienestar de las personas, pues llega a conceptualizarse como el espacio que cualquier ser humano precisa para aprender, crecer, desarrollarse y crear su propia representación del mundo (Aguilar, 2005; Estévez, Jiménez y Musitu, 2007; Urbano y Bernedo, 2016), cuestión que adquieren aún más relevancia en una etapa de constante cambio y evolución como es la adolescencia (Álvarez, 2006). Considerando que actualmente, coexisten numerosas posibilidades y variantes a la hora de intentar definir y contextualizar el término familia, emerge el interrogante entre los/as estudiosos de estas temáticas sobre si no sería más pertinente instaurar directamente el de "familias", pues ello puede suponer un estímulo para la propia aceptación de la diversidad
(Musitu y Cava, 2001) que este concepto encierra intrínsecamente. Así las cosas, y reconociendo el hecho de que la definición de "familia" se encuentra en permanente debate siendo, por tanto, extremadamente difícil optar por una definición que englobe toda la pluralidad de estructuras familiares (Musitu, Estévez y Jiménez, 2010), sí parece sin embargo advertirse cierto consenso al entender a la familia como un proyecto vital común vehiculizado a través de un proceso comunicativo e interaccional circunscrito a un marco educativo compartido y en el que se establece un fuerte compromiso emocional entre sus miembros (Corsi, 2003; Palacios y Rodrigo, 1998), siendo entonces su fin último el desarrollo integral de los sujetos que la integran (Ceballos, 2009).

De las numerosas dimensiones y variables que influyen, condicionan y configuran el contexto familiar, la adaptabilidad y la cohesión familiar desempeñan un papel fundamental al hacer 
referencia, en el caso de la cohesión, al vínculo emocional que los miembros de la familia tienen entre sí, incluyendo aspectos como los lazos familiares, la implicación y las coaliciones padres-hijos, mientras que la adaptabilidad alude a la capacidad de la familia para cambiar, contemplando cuestiones como el liderazgo, la disciplina, los roles, las reglas y la negociación (Grupo EIF, 2013). Por su parte, González et al. (2002) abordan conjuntamente ambas dimensiones delimitando la cohesión familiar al vínculo emocional y la autonomía que se da entre los miembros de la familia (incorporando aspectos como la independencia, las coaliciones, el tiempo, los espacios o la toma de decisiones) y la adaptabilidad a la habilidad que presentan las familias para modificar sus estructuras, como los roles y normas de relación, en respuesta al desarrollo evolutivo de la propia familia o al estrés que conllevan determinadas situaciones (asertividad, negociar, control, disciplina, etc.).

Así pues, estas dos dimensiones configuran, en palabras de López (2002), un eje vertebrador de las relaciones familiares al abordar el contexto familiar de forma global, permitiendo, por un lado, obtener una imagen completa de la familia y, por otro, considerar tanto lo que el sujeto percibe realmente de sus relaciones familiares como lo que estima como ideal en tales relaciones, obteniéndose así una doble perspectiva entre la información de la propia familia y del ideal de familia.

Asimismo, y conforme lo expuesto en líneas superiores, autores como Robledo y García (2009), tras realizar una revisión exhaustiva de estudios nacionales e internacionales centrados en la influencia de variables familiares y la adaptación académica, persiguen vincular los contextos familiar y escolar, corroborando la incidencia en el desarrollo escolar de aspectos vinculados al clima y funcionamiento del hogar, las percepciones y conductas de los padres hacía los hijos o la implicación de los padres en la educación (Álvarez y Martínez, 2016; Cordero, Manchón y Simancas, 2014). En definitiva y como se ha podido observar, familia y escuela, escuela y familia son dos microsistemas "condenados" a comunicarse, cooperar y entenderse en pro del éxito sociopersonal y académico de estos/as estudiantes, motivo que también nos hace traer a colación la necesaria observación y diagnóstico sobre los entornos comunitarios y espacios que trascienden el núcleo familiar (Martínez, 2011; Symeou, Martínez-González y Álvarez, 2012), máxime si, como resaltan Pegalajar y Colmenero (2013, p. 35): “cuanto más desestructurado sea el entorno sociofamiliar del alumno, mayor será la posibilidad de que desarrolle alteraciones y/o desajustes personales que dificulten su pleno proceso de adaptación social, escolar y familiar".

Puesto que en las investigaciones consultadas se observa cierta tendencia a analizar el contexto familiar de estudiantes universitarios o la influencia de la familia en los primeros años de edad (Banquerri, Méndez y Arias, 2017), en este estudio se apuesta por profundizar en el análisis del ambiente familiar en estudiantes de Secundaria (Álvarez, 2006; Martínez y Álvarez, 2005), siendo ellos mismos quienes aporten esta información con el objetivo de identificar áreas prioritarias de apoyo con las que poder planificar actuaciones encaminadas a la mejora y a la prevención centradas en la etapa de la adolescencia_Álvarez-García, Dobarro, Álvarez, Núñez y Rodríguez, 2014) que repercutan positivamente a nivel personal, familiar y, específicamente como es el caso particular de esta investigación, en la esfera académica.

Se concluye señalando que incluso en esta etapa, caracterizada por los drásticos y rápidos cambios que se producen a nivel físico, cognitivo, emocional y social (con las consecuentes ambivalencias y contradicciones hacia uno mismo y a la sociedad), el papel y el apoyo de los progenitores continúan siendo, al igual que en etapas anteriores, valiosos e irremplazables factores de protección para los hijos ante conductas de riesgo prototípicas de este estadio. Algunos de los comportamientos a los que se está aludiendo guardan relación, por ejemplo, a "la repetición de curso, el consumo de drogas y determinadas conductas agresivas como el acoso escolar" (Méndez y Cerezo, 2018) o la manifestación de una baja autoestima, desmotivación, bajo compromiso personal con el estudio, reducidas o nulas expectativas académicas y episodios de fracaso y/o riesgo de abandono prematuro de los estudios (Álvarez y Martínez, 2017). Por ello, parece conveniente apostar por investigaciones en las que se reconozca la relevancia de este agente de socialización, la familia, núcleo convivencial y de aprendizajes compartidos en los que como factores de protección sobresale el intercambio de afectos (emociones) y la comunicación, ambas piezas clave en un óptimo ajuste psicosocial en la adolescencia (Oliva, Parra, Jiménez, Sánchez-Queija y López, 2007; Rodrigo et al., 2004).

\section{Método}

\section{Participantes}

Han participado 296 estudiantes de Educación Secundaria Obligatoria (ESO), de los cuales el 51\% son chicos y el $49 \%$ chicas. Por cursos, $1^{\circ}$ de ESO queda representado por el $29,4 \%$, $2^{\circ}$ por el $20,3 \%$, $3^{\circ}$ por el $28,7 \%$ y $4^{\circ}$ por el $21,6 \%$. La edad media de los participantes es de 14,06 años (DT =1,46; moda y mediana de 14 años) y de nacionalidad mayoritaria española ( $83,2 \%$; por continentes, el 9,3\% son de América Latina, el 5,2\% de otros países de Europa, el 1,7\% de África y el 0,7\% de Asia).

En cuanto a su contexto familiar, el $61,8 \%$ vive en una familia biparental clásica (constituida por un hombre, una mujer y los hijos de ambos), el 20,1\% en una monoparental, el 11,2\% en una familia reconstituida y el 6,9\% restante indica otras opciones. Referente al nivel de estudios del padre, el 31,7\% indica estudios equivalentes a la ESO, otro 31,7\% universitarios, el 28,6\% bachillerato o Formación Profesional (FP) y únicamente el 7,9\% no cuenta con estudios reglados. En el caso de las madres, el 35,7\% cuenta con estudios universitarios, el 31,1\% cursó bachillerato o FP, el 23,9\% estudios equivalentes a la ESO y solo el 9,2\% no cuenta con estudios.

A nivel escolar, el 72,4\% nunca ha repetido un curso. Además, el 53,8\% del alumnado tiene expectativas de finalizar estudios universitarios, el 15,5\% bachillerato, el 13,8\% un ciclo formativo de grado superior, el $8 \%$ la ESO, el 6,9\% un ciclo formativo de grado medio y únicamente el $1 \%$ considera que no va a obtener un título formativo (otro $1 \%$ indica otras opciones).

\section{Instrumento}

Para medir ambas dimensiones, se ha utilizado la Escala de adaptabilidad y cohesión familiar. Esta escala destaca tanto en el contexto internacional como nacional, pues la Family Adaptability and Cohesion Evaluation Scale de Olson, Portner y Bell (1982), adaptada al contexto español por Martínez-Pampliega, Iraurgi, Galindez y Sanz (2006), sigue siendo ampliamente utilizada en disciplinas y ámbitos tan diversos como la psicología (Kluck, Dallesasse y English, 2017; Schaffer, Barak y Rassovsky, 2015), la medicina (Fernández-Castillo y Vílchez-Lara, 2016), la evaluación del contexto familiar (Villavicencio y López, 2017) o el estudio de las relaciones familiares vinculadas a lo educativo (Chávez, Calderón y Hidalgo, 2015; Iglesias y Calmet, 2015).

La escala tiene 20 ítems, utiliza una escala de respuesta tipo Likert de cuatro opciones y sus índices de validez y fiabilidad son buenos (Alfa de Cronbach de 0,89 en cohesión, 0,87 para adaptabilidad y 0,90 global). La subescala de cohesión estaría compuesta por los ítems 1, 4, 7, 9, 10, 13, 15, 17, 18 y 20; integrando la de adaptabilidad los ítems restantes: 2, 3, 5, 6, 8, 11, 
$12,14,16$ y 19. En base a los autores que han adaptado la escala al contexto español, este instrumento ha sido utilizado en miles de investigaciones a nivel internacional y en numerosas investigaciones en el contexto español. En esta investigación, la escala ha obtenido una fiabilidad de .92 , un valor considerado como "excelente" según George y Mallery (2003).

\section{Procedimiento de recogida de la información}

Dentro de este epígrafe cabe señalar que en el presente estudio se ha optado por un muestreo no probabilístico y, concretamente, un muestreo de conveniencia, también llamado fortuito o accidental, pues los casos seleccionados han sido aquellos que se encontraban más disponibles, siendo las características de este tipo de muestreo (más sencillo, rápido y económico) el motivo de su selección (Alaminos y Castejón, 2006).

El cuestionario fue administrado dentro de la jornada lectiva de forma voluntaria al alumnado aprovechando algunas de las sesiones de tutorías, contando con la presencia de al menos un investigador y el tutor del grupo en todo momento con el fin de garantizar que cualquier duda de contenido o metodológica pudiera ser resuelta. Incluyendo la explicación previa a su administración, el tiempo empleado para cumplimentar el cuestionario fue de entre 15 y 25 minutos.

Todos los cuestionarios se recogieron entre la última semana de marzo y la primera de abril de 2017. Una vez finalizada la recogida de datos se procedió a vaciar y analizar dicha información a través del programa informático SPSS 22.0.

\section{Análisis de datos}

Con el objetivo de facilitar al/la lector/a la revisión de los resultados de investigación que se expondrán en el siguiente epígrafe, cabe apuntar que, en primer lugar, se presentan los resultados descriptivos (porcentajes, medias y desviación típica) de cada ítem del cuestionario. A continuación, se efectúa un contraste de medias entre las variables de clasificación que presenta la muestra, a saber, género (hombre/mujer), nacionalidad (española/otras), curso académico agrupado en dos opciones ( $1^{\mathrm{0}}$ y $2^{\circ}$ frente a $3^{\circ}$ y $4^{\circ}$ de ESO), hecho de ser repetidor (sí/no), expectativas académicas (estudios universitarios/otros), niveles de estudios paternos y maternos (estudios universitarios/otros) y pertenencia a una estructura familiar biparental clásica $\left(\mathrm{sí} / \mathrm{no}^{1}\right)$. Las posibles diferencias significativas se han comprobado mediante el estadístico de contraste $t$ de Student para dos muestras independientes. Por su parte, el tamaño del efecto se ha calculado con el estadístico $d$ de Cohen, considerando que valores de $0<\mathrm{d}<0,20$ indican un efecto pequeño, entre $0,20<\mathrm{d}<0,50$ informan sobre un efecto mediano y $\mathrm{d}>0,50$ apunta un efecto grande (Cohen, 1988), aunque considerando que incluso un tamaño del efecto pequeño puede tener una significación práctica (Kirk, 1996). Además, se incluyen análisis predictivos utilizando la regresión lineal a través del método "paso a paso" para maximizar el conocimiento de la contribución relativa de cada proceso, con el fin de determinar el peso explicativo de unas variables sobre otras consideradas dependientes. Previamente a la ejecución de análisis de regresión se calculó la correlación de Pearson entre todas las variables predictoras, asegurando con ello que dichas correlaciones bivariadas fueran menores a 0,70 $\mathrm{y}$, en consecuencia, descartando la multicolinealidad (Tabachnick y Fidell, 1996).

\section{Resultados}

Como se observa en la Tabla 1, en general se aprecian resultados positivos, destacando cuestiones como el sentimien-

Tabla 1. Resultados descriptivos de la adaptabilidad y cohesión familiar

\begin{tabular}{|c|c|c|c|c|c|c|}
\hline Ítems & $\begin{array}{c}\text { Nada } \\
(\%)\end{array}$ & $\begin{array}{c}\text { Poco } \\
(\%)\end{array}$ & $\begin{array}{c}\text { Algo } \\
(\%)\end{array}$ & $\begin{array}{c}\text { Mucho } \\
(\%)\end{array}$ & - & Sx \\
\hline 1. Los miembros de mi familia se sienten muy cercanos unos a otros & 2 & 11,5 & 39,7 & 46,8 & 3,3 & 0,75 \\
\hline 2. Cuando hay que resolver problemas, se siguen las propuestas de los hijos & 17,5 & 44,1 & 32,5 & 5,9 & 2,3 & 0,82 \\
\hline 3. En nuestra familia la disciplina (normas, obligaciones, castigos) es justa & 4,8 & 14,4 & 45,5 & 35,3 & 3,1 & 0,82 \\
\hline 4. En mi familia asumimos las decisiones que se toman de forma conjunta como familia & 8,9 & 19,8 & 42,3 & 29 & 2,9 & 0,92 \\
\hline 5. En cuanto a la disciplina, se tiene en cuenta la opinión de los hijos & 14,8 & 33,1 & 38,6 & 13,4 & 2,5 & 0,90 \\
\hline 6. Cuando surgen problemas, negociamos para encontrar una solución & 6,8 & 23,5 & 41,8 & 27,9 & 2,9 & 0,88 \\
\hline 7. En nuestra familia hacemos cosas juntos & 5,7 & 21,6 & 38,9 & 33,8 & 3 & 0,89 \\
\hline 8. Los miembros de la familia decidimos lo que queremos libremente & 10,5 & 27,9 & 40,1 & 21,6 & 2,7 & 0,92 \\
\hline 9. En nuestra familia nos reunimos todos juntos en la misma habitación (salón, cocina) & 8,9 & 21,8 & 38,6 & 30,7 & 2,9 & 0,94 \\
\hline 10. En mi familia nos apoyamos unos a otros en los momentos difíciles & 3,7 & 10,5 & 26,8 & 59 & 3,4 & 0,82 \\
\hline 11. Los padres y los hijos hablamos juntos sobre el castigo & 26,8 & 28,2 & 30,6 & 14,4 & 2,3 & 0,99 \\
\hline 12. En nuestra familia, a todos nos resulta fácil expresar nuestra opinión & 8,5 & 23,5 & 38,2 & 29,7 & 2,9 & 0,93 \\
\hline 13. Los miembros de la familia compartimos intereses y aficiones & 8,9 & 23 & 43,6 & 24,4 & 2,8 & 0,90 \\
\hline 14. En nuestra familia se intentan nuevas formas de resolver problemas & 14,5 & 33,8 & 40,7 & 11 & 2,5 & 0,87 \\
\hline 15. A los miembros de la familia nos gusta pasar el tiempo libre juntos & 9,3 & 30,2 & 36,8 & 23,7 & 2,7 & 0,92 \\
\hline 16. Todos tenemos voz y voto en las decisiones familiares importantes & 12,7 & 26,8 & 41,2 & 19,2 & ,27 & 0,93 \\
\hline 17. En mi familia nos consultamos unos a otros las decisiones & 9,2 & 29,1 & 41,8 & 19,9 & 2,7 & 0,87 \\
\hline 18. Los miembros de la familia nos pedimos ayuda mutuamente & 7,6 & 19 & 45,2 & 28,3 & 2,9 & 0,88 \\
\hline 19. Comentamos los problemas y nos sentimos muy bien con las soluciones encontradas & 10,7 & 26,6 & 39,7 & 23,1 & 2,7 & 0,93 \\
\hline 20. La unidad familiar es una preocupación principal & 10 & 20 & 35,9 & 34,1 & 2,9 & 0,97 \\
\hline
\end{tabular}

Nota de los autores: las estructuras familiares diferentes a esta han sido catalogadas como diversidad familiar. 
to de cercanía entre los miembros de la familia, lo justa que es la disciplina, la realización de cosas juntos y el apoyo familiar en momentos de dificultad. Por el contrario, aquellos aspectos que presentan un mayor índice de mejora serían los relacionados con la disciplina y la participación de los hijos, como seguir las propuestas de estos ante la resolución de un problema, tener en cuenta su opinión en cuanto a disciplina, hablar padres e hijos sobre el castigo o intentar nuevas formas de resolver los problemas. Aunque en menor medida, también se pueden subrayar las posibilidades de mejora que brindan cuestiones como el hecho de que todos los miembros de la familia sientan que deciden libremente lo que desean, que comparten intereses y aficiones (muy vinculado a que a los miembros de la familia les guste pasar tiempo libre juntos), que velan porque todos tengan voz y voto en las decisiones importantes (relacionado con consultarse unos a otros las decisiones) o que comenten los problemas y se sientan a gusto con las soluciones propuestas.

Por otro lado, atendiendo al contraste de medias se han encontrado diferencias significativas en cuanto al género únicamente en el ítem 11 ( $p=0,035)$, hablando más sobre el castigo en los hogares de los chicos $y$, respecto a la nacionalidad, en el ítem $3(\mathrm{p}=0,039)$, percibiendo más justa la disciplina el alumnado de origen español frente al de otros países. En los restantes ítems analizados, y con el fin de comprobar la presencia de dichas diferencias significativas, se presenta como síntesis la Tabla 2, considerando las siguientes variables de clasificación: curso, ser repetidor, expectativas académicas, estudios del padre y de la madre y diversidad familiar.

Tabla 2. Diferencias significativas en adaptabilidad y cohesión familiar en las variables de clasificación

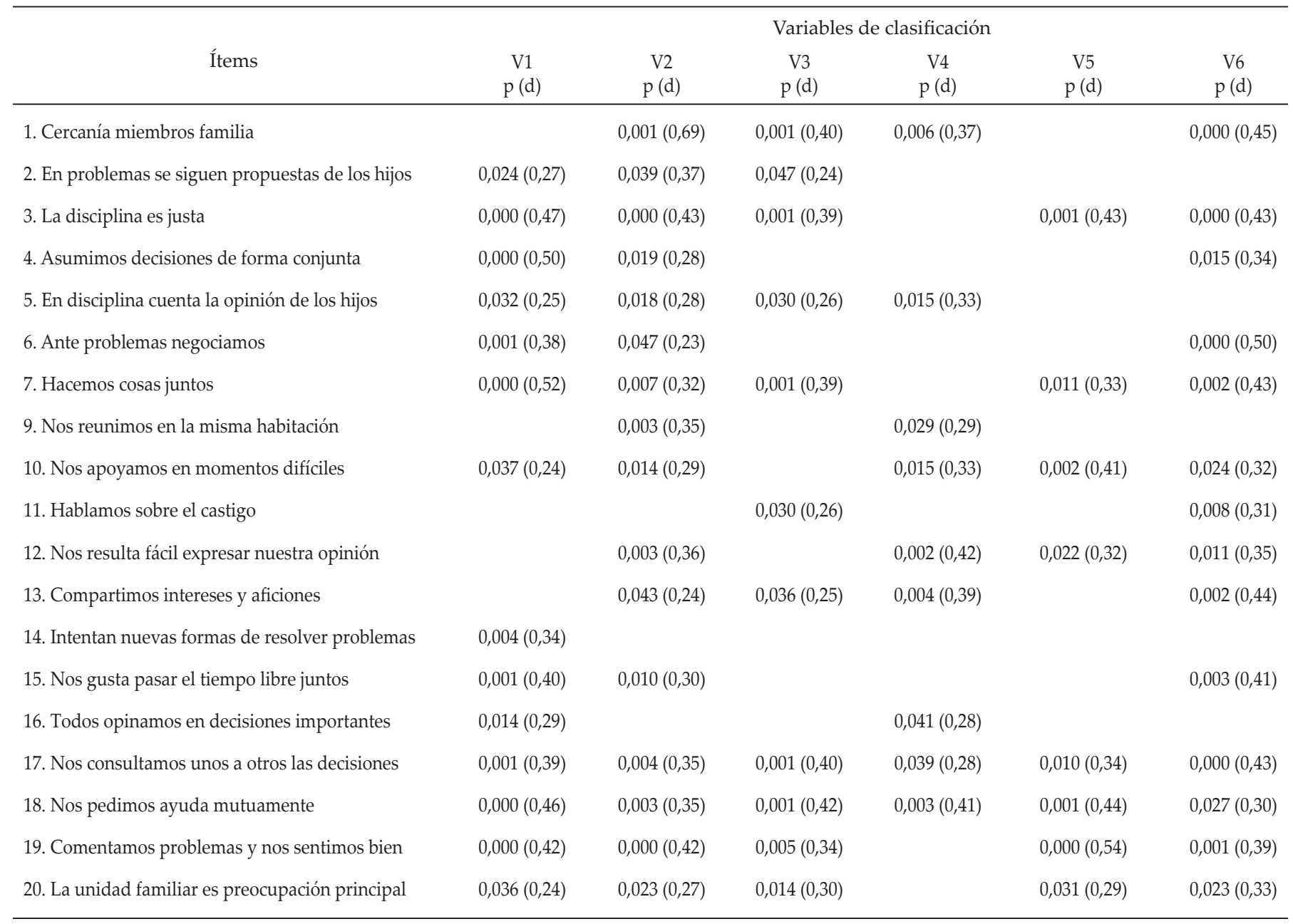

V1=Curso, V2=Ser repetidor, V3=Expectativas académicas, V4=Estudios del padre, V5=Estudios de la madre, V6=Diversidad familiar

A los datos que aporta la tabla previa debe añadirse igualmente que, en todos los casos en los que se detectan diferencias, los niveles de adaptabilidad y cohesión familiar son superiores en el alumnado de $1^{\mathrm{o}}$ y $2^{\mathrm{o}}$ de la ESO, entre el alumnado no repetidor, que tiene expectativas de cursar estudios universitarios, ambos progenitores cuentan con estudios superiores y forman parte de una familia biparental clásica. Destaca en este bloque, por un lado, que ser repetidor tenga relación con un alto número de ítems (16 de 20) y, por otro, que el alumnado más joven sea el que presenta mejor adaptabilidad y cohesión, lo que puede vincularse a que la influencia de los iguales y el desapego del núcleo familiar es más pronunciado en edades que corresponden 
a $3^{\circ}$ y $4^{\circ}$ de ESO. Además, de entre todas estas variables, destaca también la diversidad familiar (diferencias en 14 de los 20 ítems), de manera que aquellas estructuras familiares en las que existe un único progenitor o bien dentro de las familias reconstituidas, se pueden encontrar mayores dificultades, especialmente al inicio de esta situación, para lograr, por ejemplo, momentos en los que toda la familia directa del adolescente comparta espacios o actividades.

Por último, en cuanto a los análisis de regresión encaminados a intentar predecir algunas de las variables que integran ambas dimensiones, se han seleccionado cuatro variables (dos de cohesión y dos de adaptabilidad) por su relevancia a nivel teórico y porque contribuyen a sintetizar la dimensión a la que pertenecen. Atendiendo a la cohesión familiar se analizan las variables
"Los miembros de mi familia se sienten muy cercanos unos a otros" y "A los miembros de la familia nos gusta pasar el tiempo libre juntos", mientras que respecto a la adaptabilidad las seleccionadas han sido "Cuando surgen problemas, negociamos para encontrar una solución" y "Comentamos los problemas y nos sentimos muy bien con las soluciones encontradas".

La primera de ellas, referente al sentimiento de cercanía entre los miembros de la familia, queda explicada por un modelo predictivo (ver Tabla 3) integrado por dos variables que contribuyen a explicar el $40 \%$ de la varianza y entre las que destaca hacer cosas juntos. De igual modo, también parece influir el apoyo mostrado en los momentos difíciles como favorecedor del sentimiento de cercanía.

Tabla 3. Modelo predictivo de la variable: “Los miembros de mi familia se sienten muy cercanos unos a otros"

\begin{tabular}{cccc}
\hline Modelo & $\mathrm{R}^{2}$ ajustado & Beta & Sig, Cambio en F \\
1 & 0,33 & 0,42 & 0,000 \\
2 & 0,40 & 0,32 & 0,000
\end{tabular}

Predictores: En nuestra familia hacemos cosas juntos, En mi familia nos apoyamos unos a otros en los momentos difíciles

Más centrada en la dimensión de adaptabilidad, resulta de interés conocer que lleva a las familias a negociar cuando surge un problema. Con el fin de predecir esta variable se ha generado un modelo predictivo (ver Tabla 4) que permite explicar el 37\% de la varianza y que está compuesto por tres variables que hacen referencia a contar con la opinión de los hijos en cuanto a disciplina, comentar los problemas y sentirse bien con las soluciones encontradas, y considerar que la disciplina es justa.

Tabla 4. Modelo predictivo de la variable: “Cuando surgen problemas, negociamos para encontrar una solución"

\begin{tabular}{cccc}
\hline Modelo & $\mathrm{R}^{2}$ ajustado & Beta & Sig, Cambio en F \\
1 & 0,26 & 0,33 & 0,000 \\
2 & 0,35 & 0,26 & 0,000 \\
3 & 0,37 & 0,19 & 0,001 \\
Predictores: En cuanto a la disciplina, se tiene en cuenta la opinión de los hijos, Comentamos los problemas yos senti- \\
mos muy bien con las soluciones encontradas, En nuestra familia la disciplina (normas, obligaciones, castigos) es justa
\end{tabular}

En tercer lugar, otro elemento relevante es el tiempo que se pasa en familia y, concretamente, constatar si a los miembros de la familia les gusta pasar su tiempo libre juntos. Para predecir esta cuestión se ha obtenido un modelo (ver Tabla 5) constituido por tres variables que logran explicar el $45 \%$ de la varianza de esta variable independiente. En este caso, además de incluirse variables directamente relacionadas como que se hagan cosas juntos como familia, también aparecen otros aspectos como comentar los problemas (sintiéndose bien con las soluciones encontradas) y percibir que todos tienen voz y voto a la hora de adoptar decisiones familiares importantes.

Tabla 5. Modelo predictivo de la variable: "A los miembros de la familia nos gusta pasar el tiempo libre juntos"

\begin{tabular}{cccc}
\hline Modelo & $\mathrm{R}^{2}$ ajustado & Beta & Sig, Cambio en F \\
1 & 0,34 & 0,36 & 0,000 \\
2 & 0,42 & 0,28 & 0,000 \\
3 & 0,45 & 0,21 & 0,000
\end{tabular}

Predictores: En nuestra familia hacemos cosas juntos, Comentamos los problemas y nos sentimos muy bien con las soluciones encontradas, Todos tenemos voz y voto en las decisiones familiares importantes 
Finalmente, se ha considerado la necesidad de intentar predecir la variable referente a comentar los problemas y sentirse bien con las soluciones encontradas, pues ha contribuido a la explicación de otras dos variables y guarda relación con el tema de la disciplina, una de las cuestiones que mostraban mayor margen de mejora. Con esta finalidad, los análisis ejecutados arrojan un modelo predictivo (ver Tabla 6) algo más complejo al estar compuesto por cinco variables que alcanzan a explicar el $49 \%$ de la varianza de la variable independiente cuyo estudio se está abordando. En esta ocasión, las variables que actúan como predictores hacen alusión a pedir ayuda entre los miembros de la familia, negociar los problemas para buscar una solución, que resulte fácil expresar opiniones, que se intenten nuevas formas de resolver los problemas y asumir las decisiones que se toman de forma conjunta como familia.

Tabla 6. Modelo predictivo de la variable: "Comentamos los problemas y nos sentimos muy bien con las soluciones encontradas"

\begin{tabular}{|c|c|c|c|}
\hline Modelo & $\mathrm{R}^{2}$ ajustado & Beta & Sig, Cambio en F \\
\hline 1 & 0,31 & 0,21 & 0,000 \\
\hline 2 & 0,39 & 0,17 & 0,000 \\
\hline 3 & 0,43 & 0,19 & 0,000 \\
\hline 4 & 0,46 & 0,21 & 0,000 \\
\hline \multicolumn{4}{|c|}{$\begin{array}{l}\text { Predictores: Los miembros de la familia nos pedimos ayuda mutuamente, Cuando surgen problemas, negociamos para } \\
\text { encontrar una solución, En nuestra familia, a todos nos resulta fácil expresar nuestra opinión, En nuestra familia se } \\
\text { intentan nuevas formas de resolver problemas, En mi familia asumimos las decisiones que se toman de forma conjunta } \\
\text { como familia }\end{array}$} \\
\hline
\end{tabular}

\section{Discusión y conclusiones}

Las funciones de naturaleza educativa y de socialización otorgan a la familia una marcada dimensión pública al contribuir a la formación de ciudadanos y a la generación de capital social (Martínez y Becedóniz, 2009). En innumerables ocasiones, la atención suele centrarse casi en exclusiva en el comportamiento de los hijos, sobre todo si se tiene en cuenta que en psiquiatría infantil y adolescente los problemas de comportamiento son el motivo más frecuente de consulta (Gamazo, Díaz-Caneja Greciano, Rapado y Soutullo, 2007). Sin embargo, si se quiere entender realmente la situación y el contexto en el que viven los adolescentes, es necesario sumar el estudio del contexto familiar al análisis de su comportamiento, pues las prácticas de crianza de los padres se relacionan con los problemas de conducta de los hijos. Por ejemplo, cuando se utilizan prácticas de crianza inadecuadas (como el afecto negativo, los castigos físicos o el control autoritario), los hijos presentan mayor probabilidad de manifestar este tipo conductas (Ramírez, 2002).

En esta línea, al profundizar en el estudio de la adaptabilidad y cohesión familiar, y a pesar de encontrar resultados generales positivos, se debe trabajar para que la disciplina sea justa y favorecer la participación de los hijos en las decisiones familiares. Para ello, se pueden incorporar sus propuestas a la resolución de los conflictos, hablar más sobre los castigos o conocer su opinión sobre la disciplina, lo que contribuirá a mejorar aspectos como que todos consideren que se pueden comentar los problemas y se sientan bien con las soluciones encontradas.

De los beneficios que puede conllevar la adquisición de habilidades para una adecuada resolución de conflictos dentro del sistema familiar, destaca la propia relación entre los progenitores. Cuando el conflicto es una constante entre una pareja ambos sufren, agravándose la situación cuando existen hijos en el hogar $\mathrm{y}$, especialmente, cuando estos se encuentran o alcanzan la etapa de la adolescencia (Rodrigo et al., 2004). Diversas investigaciones han contribuido a identificar el interparental como un condicionante negativo para el desarrollo de los niños, pues aquellos que se ven expuestos con frecuencia e intensidad a esta situación reportan más riesgo de presentar dificultades como problemas de conducta, desobediencia, falta de control, menor integración en el grupo de iguales y peores resultados académicos (Cantón, Corté y Justicia, 2002; Golombok, 2006; Symeou, Martínez-González y Álvarez, 2012).

Por otro lado, como recogen Martínez, Pérez y Álvarez (2007), un aspecto realmente complejo para las familias es establecer normas y límites a los hijos, así como delimitar y aplicar consecuencias que sean lógicas, consensuadas y proporcionadas cuando se incumple lo establecido. A pesar de ello, cuando los padres interiorizan esta necesidad y adquirieren las competencias necesarias para ello, experimentan los beneficios del establecimiento y cumplimiento de los límites y normas, pues los hijos van adquiriendo referencias consistentes de hasta dónde pueden llegar con sus demandas y actuaciones. Además, la puesta en práctica de estas competencias conlleva, por parte de los niños, el desarrollo progresivo de la tolerancia a la frustración y de habilidades de autorregulación (Musitu, Estévez y Jiménez, 2010), competencias imprescindibles si se quiere alcanzar una adecuada adaptación e integración social desde los primeros años de vida.

En esta línea argumental, Oliva (2006) señala que cuando los padres son afectuosos, comunicativos y contribuyen a que sus hijos sean autónomos, los adolescentes se ven muy favorecidos, alejando así de la visión negativa que suele asociarse a las relaciones entre padres e hijos adolescentes. De igual modo, las investigaciones llevadas a cabo por Morales, Félix, Rosas, López y Nieto (2015) indican que, cuando los padres son capaces de transmitir unas instrucciones claras, establecen normas y límites, optan por la resolución de problemas y una interacción social positiva (características asociadas a un estilo educativo democrático), desarrollan estilos de crianza que correlacionan positivamente con una menor tasa de aspectos como la conducta negativista desafiante y la agresión infantil en los hijos. En este punto, ya es posible vincular el contexto familiar, y los aprendizajes que en él se llevan a cabo, con el bienestar y el comportamiento de los niños en contextos como el escolar, así como con otras variables como la autorregulación o la tolerancia a la frustración.

Aunque, desde el modelo preventivo por el que se debería apostar en las actuaciones socioeducativas todo el alumnado y 
sus familias son susceptibles de participar en cualquier acción encaminada a potenciar las habilidades que ya tienen adquiridas y reducir las dificultades que puedan presentar, en este caso, los contextos familiares que requieren una actuación más acuciante son los del alumnado de cursos superiores ( $3^{\circ}$ y $4^{\circ}$ de la ESO), aquel que es repetidor, que tiene expectativas académicas más bajas, sus progenitores cuentan con niveles educativos más bajos y presentan mayor diversidad familiar. Este hallazgo también ha sido corroborado en investigaciones como las desarrolladas por Álvarez (2006) y Álvarez y Martínez (2017).

Por último, se desea hacer constancia de algunas limitaciones del presente estudio, Así, cabe comentar primeramente que la información se ha obtenido por medio de autoinformes pues, aunque en ocasiones son preferibles a otras técnicas como la observación (más costosa y difícil de organizar) o las entrevistas (carecen generalmente de baremos), suelen asociarse a problemas de sesgo vinculados a la deseabilidad social (Fernández-Montalvo y Echeburrúa, 2006). Esta última limitación conecta directamente con las futuras líneas de trabajo de modo que parece interesante incorporar otros instrumentos de recogida de información de naturaleza cualitativa como entrevistas o grupos de discusión entre los propios informantes, lo que permitiría ampliar y complementar los datos cuantitativos obtenidos y, en consecuencia, conferir una mayor validez a los resultados aquí presentados. Asimismo, otra de las sugerencias alude a una posible ampliación de la muestra de estudio, lo que contribuiría a incrementar la diversidad entre los propios sujetos y su entorno (ciudades, entornos urbanos, zonas geográficas de interior o costeras, etc.,). De igual modo, se podrían considerar como escenarios o realidades informantes otras etapas educativas (como estudiantes de Formación Profesional, Bachillerato o Universidad) con el fin de conocer la tendencia que siguen los resultados en diferentes niveles educativos y etapas evolutivas, valorando con ello qué edades requieren una mayor atención y qué acciones específicas son necesarias para cada edad.

En síntesis, y atendiendo a las ideas y argumentaciones expresadas en líneas superiores, se entiende que una de las principales aportaciones de este artículo radica en subrayar nuevamente, en esta ocasión desde la voz de un colectivo adolescente, el valor del ambiente, rutinas familiares $y$, en consecuencia, estilos de educación parental (Martínez y Becedóniz, 2009) como factores de promoción de conductas autorregulatorias en los menores (González-Pienda, Núñez, González-Pumariega, Álvarez, Roces y García, 2002), y de protección ante situaciones de desestructuración familiar, riesgo de exclusión social o abandono escolar. En consonancia con las líneas de investigación preferente de los autores de este artículo y del grupo de investigación al que pertenecen, y pensando en una práctica concreta, se apuesta por el diseño e implementación de programas de formación o capacitación parental en los que tanto los progenitores como su descendencia participen conjuntamente abordando los contenidos que el Consejo de Europa recoge en su Recomendación 2006/19 sobre el ejercicio positivo del rol parental (Álvarez y Martínez, 2016, 2017; Epstein, 2011; Rodrigo, Máiquez, Martín, Byrne y Rodríguez, 2015).

\section{Referencias bibliográficas}

Aguilar, M.C. (2005). Las prácticas educativas en el ámbito familiar, En L, M, Naya Garmandia y P, Dávila Balsera (Eds,). La infancia en la historia: espacios y representaciones (pp, 73-82), San Sebastián: Erein.

Alaminos, A. y Castejón, J.L. (2006). Elaboración, análisis e interpretación de encuestas, cuestionarios y escalas de opinión, Alicante: Universidad de Alicante,
Álvarez, L. (2006). Familia y abandono escolar, Madrid: Cinca.

Álvarez-García, D., Dobarro, A., Álvarez, L., Núñez, J.C. y Rodríguez, C. (2014). La violencia escolar en los centros de educación secundaria de Asturias desde la perspectiva del alumnado, Educación XX1, 17(2), 337-360, doi: 10,5944/educxx1,17,2,11494.

Álvarez, L., y Martínez, R.A. (2016). Cooperación entre las Familias y los Centros Escolares como Medida Preventiva del Fracaso y del Riesgo de Abandono Escolar en Adolescentes, Revista Latinoamericana de Educación Inclusiva, 10(1), 175192.

Álvarez, L., y Martínez, R.A. (2017). Review on School and Family Partnership, Shared Responsibility as Quality Indicator, en González, J.L., Bernardo, A., Núñez, J.C. and Rodríguez, C. (2017), Factors Affecting Academic Performance (pp,121-140), New York: Nova Science Publisher.

Banquerri, M., Méndez, M., y Arias, J.L. (2017). Parental support in early childhood and its importance for the brain, en González, J.L., Bernardo, A., Núñez, J.C., y Rodríguez, C. (2017), Factors Affecting Academic Performance (pp,103-119), New York: Nova Science Publisher.

Cantón, J., Cortés, M.R. y Justicia, M.D. (2002). Las consecuencias del divorcio en los hijos, Psicopatología Clínica, Legal y Forense, 2(3), 47-66.

Ceballos, M. (2009). La educación formal de los hijos e hijas de familias homoparentales: familia y escuela a contracorriente, Aula Abierta, 37(1), 67-78,

Cordero, J.M., Manchón, C. y Simancas, R. (2014). La repetición de curso y sus factores condicionantes en España, Revista de Educación, 365, 12-37, doi: 10,4438/1988-592X-RE- 2014-365263.

Chávez, G., Calderón, S.V. y Hidalgo, M. (2015). Funcionamiento familiar y estilos atribucionales en estudiantes de Psicología, Revista de Psicología, 10(1), 111-124.

Cohen, J. (1988). Statistical power analysis for the behavioral science ( $2^{\mathrm{a}}$ ed,). Hillsdale, NJ: Lawrence Erlbaum Associates.

Corsi, J. (2003). Maltrato y abuso en el ámbito doméstico, Fundamentos teóricos para el estudio de la violencia en las relaciones familiares, Buenos Aires: Editorial Piados.

Epstein, J.L. (2011). School, family and community partnerships, preparing educators and improving schools, Philadelphia, PA: Westview Press.

Estévez, E., Jiménez, T. y Musitu, G. (2007). Relaciones entre padres e hijos adolescentes, Valencia: Nau Llibres.

Fernández-Castillo, A. y Vílchez-Lara, M.J. (2016). La ansiedad y su asociación con elementos de funcionamiento familiar en los padres de niños atendidos en servicios de urgencias pediátricos andaluces, Emergencias, 28(4), 239-242.

Fernández-Montalvo, J. y Echeburrúa, E. (2006). Uso y abuso de los autoinformes en la evaluación de los trastornos de personalidad, Revista de Psicopatología y Psicología Clínica, 11(1), 1-12.

Gamazo, P., Díaz-Caneja, A., Rapado, M. y Soutullo, C. (2007). Aplicación de un programa de manejo de conducta para padres de hijos con problemas de comportamiento, Anales de psiquiatría, 23(4), 172-176.

George, D. y Mallery, P. (2003). SPSS for Windows step by step: A simple guide and reference, 11,0 (4th ed,), Boston: Allyn y Bacon.

Golombok, S. (2006). Modelos de familia ¿Qué es lo que de verdad cuenta?, Barcelona: Graó,

González-Pienda, J. A., Núñez, J.C., González-Pumariega, S., Álvarez, L., Roces, C. y García, M. (2002). A structural equation model of parental involvement, motivational and aptitudinal 
characteristics, and academic achievement, The Journal of Experimental Education, 70(3), 257-287.

Grupo EIF (2013). Manual de instrumentos de evaluación familiar, Madrid: CCS,

Iglesias, L. y Calmet, V.V. (2015). Factores psicológicos, sociales y demográficos asociados al rendimiento académico en estudiantes universitarios, Revista de Psicología, 12(1), 216-236.

Kirk, R.E. (1996). Practical significance: A concept whose time has come, Educational y Psychological Measurement, 56, 746759.

Kluck, A.S., Dallesasse, S. y English, E.M. (2017). Family Relations and Psychopathology: Examining Depressive and Bulimic Symptomatology, Child Psychiatry y Human Development 48(5), 818-827, doi: 10,1007/s10578-016-0705-9.

López, S. (2002). El FACES II en la evaluación de la cohesión y la adaptabilidad familiar, Psicothema, 14(1), 159-166.

Martínez, J.S. (2011). Género y origen social: diferencias grandes en fracaso escolar administrativo y bajas en rendimiento escolar, Revista de la Asociación de Sociología de la Educación, 4(3), 270-285.

Martínez, R.A. y Álvarez, L. (2005). Fracaso y abandono escolar en la Educación Secundaria Obligatoria, Implicación de la familia y los centros escolares, Aula Abierta, 85, 127-146.

Martínez, R.A. y Becedóniz, C.M. (2009). Orientación Educativa para la Vida Familiar como Medida de Apoyo para el Desempeño de la Parentalidad Positiva, Intervención Psicosocial, 18(2), 97-112.

Martínez, R.A., Pérez, M.H. y Álvarez, L. (2007). Estrategias para prevenir y afrontar conflictos en las relaciones familiares (padres e hijos), Madrid: Ministerio de Trabajo y Asuntos Sociales, Colección Observatorio de Infancia.

Martínez-Pampliega, A., Iraurgi, I., Galíndez, E., y Sanz, M. (2006). Family Adaptability and Cohesion Evaluation Scale (FACES), Desarrollo de una versión de 20 ítems en español, International Journal of Clinical and Health Psychology, 6(2), 317338.

Méndez, I. y Cerezo, F. (2018). La repetición escolar en educación secundaria y factores de riesgo asociados, Educación XX1, 21(1), 41-62, doi: 10,5944/educXX1,13717.

Morales, S., Félix, V., Rosas, M., López, F., y Nieto, J. (2015). Prácticas de crianza asociadas al comportamiento negativista desafiante y de agresión infantil, Avances en psicología latinoamericana, 33(1), 57-76.

Musitu, G, y Cava, M.J. (2001). La familia y la educación, Barcelona: Octaedro.
Musitu, G., Estévez, E., y Jiménez, T.I. (2010). Funcionamiento familiar, convivencia y ajuste en hijos adolescentes, Madrid: Fundación Acción Familiar.

Oliva, A. (2006). Relaciones familiares y desarrollo adolescente, Anuario de psicología, 37(3), 209-223.

Oliva, A., Parra, A., Sánchez, I. y López, F. (2007). Estilos educativos materno y paterno: evaluación y relación con el ajuste adolescente, Anales de psicología, 23(1), 49-56.

Olson, D.H., Portner, J. y Bell, R.Q. (1982). FACES II, St, Paul, MN: University of Minnesota,

Palacios, J. y Rodrigo, M.J. (1998). La familia como contexto de desarrollo humano, En M, J, Rodrigo y J, Palacios (Eds,), Familia y desarrollo humano (pp, 25-44), Madrid: Alianza Editorial.

Ramírez, M.A. (2002). Prácticas de crianza de riesgo y problemas de conducta en los hijos, Apuntes de psicología, 20(2), 7-15.

Robledo, P. y García, J.N. (2009). El entorno familiar y su influencia en el rendimiento académico de los alumnos con dificultades de aprendizaje: revisión de estudios empíricos, Aula Abierta, 37(1), 117-128.

Rodrigo, M.J., Máiquez, M.L., García, M., Mendoza, R., Rubio, A., Martínez, A., y Martín, J.C. (2004). Relaciones padres-hijos y estilos de vida en la adolescencia, Psicothema, 16(2), 203210.

Rodrigo, M.J., Máiquez, M.L., Martín, J.C., Byrne, S. y Rodríguez, B. (2015). Manual práctico de parentalidad positiva, Madrid: Síntesis.

Schaffer, Y., Barak, O., y Rassovsky, Y. (2015). Social Perception in Borderline Personality Disorder: The Role of Context, Journal of Personality Disorders, 29 (2), 275-288, doi: 10,1521/ pedi_2013_27_090.

Symeou, L., Martínez-González, R.A. y Álvarez Blanco, L. (2012). Dropping out of high school in Cyprus: Do parents and the family matter? International Journal of Adolescence and Youth, 17(4) 1-19, doi:10,1080/02673843,2012,717899.

Tabachnick, B.G. y Fidell, L.S. (1996). Using multivariate statistics ( $\left.3^{\mathrm{a}} \mathrm{Ed},\right)$, New York: Harper Collins College Publisher.

Urbino, A. y Berne do, I. M. (2016). Conocimiento y predisposición sobre el acogimiento familiar en el alumnado universitario de Málaga, Revista de Investigación en Educación, 14(1), 14-30.

Villavicencio, C. y López, S. (2017). Presencia de la discapacidad intelectual en la familia, afrontamiento de las madres, Fides et Ratio-Revista de Difusión cultural y científica de la Universidad La Salle en Bolivia, 14(14), 99-112. 